\title{
La Universidad de los Andes y las Cooperativas
}

\author{
Alberto García Müller \\ amuller@ula.ve
}

Recibido: 20.04 .08

Aceptado: 27.05.08

Sumario: 1. El Centro de Estudios Cooperativos Mérida (CEDECOM), -2. Los Estudios de Derecho Cooperativo,-3. El Programa de Estudios Abiertos en Desarrollo Social (PEADS),-4. El Centro de Investigaciones Jurídicas, - 5. La Cooperativa de la Universidad de los Andes (Coopula), -6. El Centro de Investigaciones para el Desarrollo Integral Sustentable (CIDIS) de la Universidad de los Andes,-7. El Instituto Iberoamericano de Derecho y Reforma Agraria (IIDARA), - 8. El Técnico Superior en cooperativismo y economía social,-9. Conclusiones,-10. Referencias

Resumen: Este trabajo presenta un resumen de las largas (45 años) variadas y fructíferas relaciones entre la Universidad de los Andes (ULA) de Venezuela (Universidad pública autónoma con sede en la ciudad de Mérida) con las cooperativas, en especial, en el área de la capacitación, con algunas actividades de asesoría y de investigación aplicada al sector. Igualmente, reseña los programas en ejecución y los proyectos a desarrollar en los próximos años.

Palabras clave: centro, educación, cooperativa, Universidad de los Andes, Mérida

\section{El Centro de Estudios Cooperativos Mérida (CEDECOM)}

La Universidad de los Andes fue la pionera en el país en materia de educación cooperativa, por lo menos en el período posterior al advenimiento de la democracia representativa a partir de 1960.

En abril de 1963, con la participación de los ministerios del Trabajo y de Agricultura y Cría, de la Corporación pública de Desarrollo Regional (Corpoandes) y de la Universidad de los Andes, se funda en la Facultad de Economía el Centro de Estudios Cooperativos Mérida (Cedecom) bajo la dirección de los expertos venezolanos graduados en el Instituto Coady de Canadá, Pbro. Jesús E. Thielen (había promovido las primeras cooperativas de Ahorro y Crédito en la Península de Paraguana, Estado 
Falcón, a comienzos de 1960), Dr. Kevin Le Morvan (experto de origen inglés) y Pbro. Ricardo Silgeros.

El Centro tenía por objetivos la promoción de cooperativas y la capacitación de sus integrantes por medio de cursillos; el asesoramiento técnico de las cooperativas en funcionamiento y la formación y capacitación social y técnica de los dirigentes del movimiento cooperativo.

Aun cuando una parte sustancial de sus recursos provenían del Ministerio de Agricultura, los programas de enseñanza estaban orientados hacia la cooperación de tipo urbano preconizada por la «Credit Union National Association» (CUNA) de los Estados Unidos de América.

La enseñanza impartida hacía hincapié en la historia tradicional del cooperativismo (Rochdale) exigiendo la aplicación rigurosa de los principios universales de la cooperación. Así mismo, se orientaba a la enseñanza práctica de la administración y contabilidad de las cooperativas, según los moldes de CUNA. Para Texier (1969-70) los programas de enseñanza eran dirigidos hacia la cooperación de crédito tipo urbano y eran demasiado generales («no dan formación especializada»).

La Confederación Nacional de Cooperativas confió la la formación de sus agentes y dirigentes — de origen urbano- al Centro de Mérida, que también se encargó de la instrucción de los voluntarios del Cuerpo de Paz; del personal de la Federación de Cooperativas de Ahorro y Crédito de Venezuela, así como de varias instituciones privadas interesadas en el cooperativismo. En 1964, la Universidad de Tel-Aviv, con la colaboración de la Organización de Estados Américanos y del Gobierno venezolano, dictó un curso intensivo para la formación de administradores de cooperativas.

El Centro de Estudios Cooperativos tuvo un rol preponderante en el país, especialmente en la zona andina y de los altos llanos occidentales: asumió la fundación de 47 cooperativas —especialmente de ahorro y crédito y de comercialización agrícola- así fue responsable de la formación de cuadros dirigentes (2380) y la asistencia técnica a las cooperativas.

Las cooperativas de la zona de Mérida hicieron lo posible para financiar las actividades del Centro, destinando el $20 \%$ de sus fondos de educación para el mismo. Se creó, además, un departamento encargado de llevar por contrato la contabilidad centralizada de todas las cooperativas, simplificándola y teniéndola al día.

Hacia 1970 el Centro desapareció por haber perdido el apoyo financiero tanto de la Universidad como del Gobierno nacional (el Ministerio de Agricultura le redujo los aportes presupuestarios, porqué los extensionistas agrícolas pagados por este eran utilizados para la promoción de cooperativas urbanas, lo que ocasionó una gradual pérdida de interés de 
su parte) y por una aplicación dogmática de la nueva ley de cooperativas de 1967 que lo obligó a separarse de la Universidad de los Andes.

De tal manera que el auge y declinación del cooperativismo, particularmente de ahorro y crédito en la región andina, donde tuvo su enclave de funcionamiento el Centro puede atribuirse — en gran medida - a la suerte del mismo, pues de 43 cooperativas que existían y eran asesoradas por Cedecom en 1964, quedaban 14 en 1970, lo que representa una caída de 29 cooperativas, de acuerdo a las estadísticas de Sunacoop de ese año.

\section{Los Estudios de Derecho Cooperativo}

En 1967, la Facultad de Derecho asume la organización del I Congreso Continental de Derecho Cooperativo que marca un hito histórico en el desarrollo del Derecho Cooperativo en América Latina. Se realiza en Mérida, en noviembre de 1969, en la sede de la Asociación de profesores universitarios. Las memorias del Congreso fueron publicadas por la Universidad en una edición de primera calidad.

En 1973 se abre el primer seminario sobre legislación cooperativa y los primeros trabajos de investigación jurídica en la materia, a cargo de un profesor que había sido enviado por la Universidad a Francia a estudiar cooperativismo en el célebre Colegio Cooperativo dirigido por Henry Desroche.

En 1974 el Derecho Cooperativo se inicia como materia optativa para los estudiantes del último año de la Escuela de Derecho dentro de una de las menciones que podían seleccionar los estudiantes; asignatura que permaneció en el pensum hasta 1978, cuando una deplorable revisión del currículo de la Escuela, eliminó las opciones y las materias optativas. En 1982 se reabre el Seminario especializado en legislación de cooperativas, el que se continúa hasta el presente, ampliado al análisis de la economía social o solidaria.

Desde el año 1.990 se ha mantenido una línea de investigación en Derecho Cooperativo reflejado en 4 proyectos de investigación sucesivos en la materia, con financiamiento de la instancia universitaria de apoyo a la investigación (CDCHT). Esta línea de investigación ha producido más de 30 artículos publicados en revistas especializadas en el ámbito iberoamericano. Igualmente, ha permitido participar con ponencias en múltiples eventos nacionales e internacionales relacionados con la materia.

Adicionalmente, se realizaron trabajos de sistematización jurisprudencial y de doctrina cooperativa para la Autoridad Administrativa de Aplicación, la Superintendencia Nacional de Cooperativas de Venezuela 
(Sunacoop). De igual forma, se elaboraron anteproyectos de leyes de Economía Social o Solidaria para Colombia, México y Venezuela, lo mismo que se revisó el proyecto de Código Cooperativo de Puerto Rico, de la ley de trabajo asociado de Colombia y de la ley de cooperativas del Ecuador.

\section{El Programa de Estudios Abiertos en Desarrollo Social (PEADS)}

Creado en 1980 en la Facultad de Derecho bajo la dirección del Presbitero Gerardo Koening (de origen holandés) se dedicó a la formación de promotores en desarrollo social, mediante la escolarización de dirigentes sindicales, vecinales y cooperativistas. Por varios años se desarrollaron cátedras sobre diversos tópicos del cooperativismo, lo mismo que alguna investigación de campo sobre el particular.

Sin embargo, como en el caso del Centro de Estudios Cooperativos, la mayor parte de los recursos financieros debieron ser obtenidos por recursos propios, dado que el aporte de la Universidad se limitó a pagar un personal administrativo mínimo, a todas luces insuficientes para las tareas asumidas.

En este período se publicaron algunos trabajos sobre cooperativismo, más bien orientados a la divulgación y extensión: Cómo Organizar y Manejar Cooperativas; Comercialización Cooperativa; los Símbolos del Cooperativismo.

En 1983 se pasó a la apertura internacional, cuando se iniciaron cursos de nivel latinoamericano con la participación del Instituto Coady de la Universidad Saint Francis Xavier de Antígonish, Nueva Escocia, Canadá, Sin embargo, a los 3 años el programa tuvo que se transferido al Centro de Investigaciones Jurídicas de la Facultad por haberse presentado serios conflictos internos.

EI PEADS aun incorpora en sus programas de formación de promotores sociales el tema de las cooperativas, lo mismo que en sus recientes responsabilidades de la coordinación del Servicio Comunitario Estudiantil de la Facultad, obligatorio para egresar de la Universidad.

\section{El Centro de Investigaciones Jurídicas}

De 1985 a 1988, el Centro de Investigaciones Jurídicas asumió la codirección del Curso Internacional de Desarrollo Social con el patrocinio del Instituto Coady de la Universidad de San Francisco Javier de Canadá y de la Confederación Latinoamericana de Cooperativas y Mutuales de 
Trabajadores (Colacot) con sede en Bogotá, Colombia, hasta que, por cambio de política de la Facultad, dicho curso tuvo que ser transferido a la Universidad Cooperativa de San Gil, Colombia, donde duró 6 años más.

Igualmente, prestó colaboración en la asesoría al Programa de Cooperativismo Escolar de la Dirección de Cultura y Extensión de la Universidad y la Dirección de Educación del Estado Mérida. Este proyecto impulsó la formación de más de 300 docentes de la escuela básica rural en materia de cooperativismo, de manera que pudiesen incorporar al desarrollo del currículo escolar tal objetivo.

Además, se dictaron cursos en todo el país sobre Organización y Manejo de Cooperativas y de Cajas de Ahorro (cooperativas de crédito de vínculo cerrado), y se asumió la recopilación de la Jurisprudencia nacional en la materia, para su consulta por medios electrónicos.

El financiamiento de la mayor parte de sus actividades provino de aportes del Instituto Coady, así como del pago de matrículas por los participantes de los cursos. No se logró constituir un equipo profesional permanente, lo que aunado a decisiones de las autoridades universitarias se abandonaron todas las actividades emprendidas.

\section{La Cooperativa de la Universidad de los Andes (Coopula)}

En la década de los 80 un grupo de profesores, estudiantes y trabajadores, primero de la Facultad de Humanidades y luego en otras facultades y en el Núcleo de la Universidad en el vecino Estado Trujillo, constituyeron como actividad pedagógica accesoria, una cooperativa que desarrolló actividades para la ocupación laboral de algunos estudiantes (explotación comercial de cafetines en zonas universitarias). Con la ayuda del rectorado Coopula trató (vía fusión) de recuperar una antigua cooperativa de consumo de la ciudad (Cooperativa 5 Águilas Blancas) que años atrás había sido irregularmente disuelta administrativamente (mas no liquidada) propósito que no pudo ser logrado por obstáculos burocráticos de toda índole.

\section{El Centro de Investigaciones para el Desarrollo Integral Sustentable (CIDIS) de la Universidad de los Andes}

El CIDIS que funciona en el Núcleo Universitario Rafael Rangel del Estado Trujillo a partir de mediados de los noventa ha venido implementado actividades de capacitación en la materia: cursos, jornadas, semina- 
rios, y desarrollado varias cohortes de un Diplomado en cooperativismo y economía social.

Es de destacar que desde el año 2.004 asume la edición de la Revista Venezolana de Economía Social (Cayapa) órgano del capítulo venezolano del Ciriec, con el apoyo financiero del Consejo de Desarrollo Científico y Humanístico de la Universidad y que ha logrado mantenerse hasta el momento, llevando 14 números consecutivos, lo que -en Venezuela - significa un esfuerzo extraordinario.

\section{El Instituto Iberoamericano de Derecho y Reforma Agraria (IIDARA)}

A partir del año 2004 el IIDARA, adscrito a la Facultad de Ciencias Jurídicas y Políticas de la Universidad que había venido declinando por la enfermedad y posterior muerte de su director-fundador después de casi 30 años de fecunda labor en el campo del Derecho Agrario, y frente a una auténtica emergencia nacional en materia cooperativa (en el país, como programa de gobierno, se pasó de mil a cerca de «doscientas mil» cooperativas legalizadas en 5 años) el Instituto decidió dedicarse con mayor hincapié a las actividades relacionadas con estas.

El Instituto utiliza algunos espacios físicos de la Facultad y equipos de computación financiados por la Universidad. Cuenta con 3 personas pagadas por la misma; el resto del equipo (6 personas más) debe ser financiado por ingresos propios (lo mismo que los gastos para insumos y desplazamientos) hecho este que entraba notablemente la productividad académica, además que impide la estabilidad del personal.

\section{a) En el área de la investigación aplicada}

Se han elaborado 25 módulos especializados sobre tópicos cooperativos, de los que 7 han sido publicados por editoriales nacionales y otros 3 se encuentran en trámite de hacerlo. Además, se ha logrado la publicación de 10 artículos científicos en revistas nacionales e internacionales, y la presentación de ponencias en congresos y seminarios nacionales e internacionales sobre la materia.

A partir del año 2.000, se ha venido desarrollando el Curso de Instituciones de Derecho Cooperativo, Mutual y Solidario, de forma semipresencial a distancia, que ha sido dictado por 3 veces en Venezuela, una en Cuba con la Universidad de Pinar del Río, y otra en México, con el auspicio de la Comisión de Fomento Cooperativo de la Cámara de Diputados del Honorable Congreso de la Unión. 
En este momento se encuentra en etapa final de revisión (con la colaboración del experto argentino Dr. Alfredo Moirano) para publicación por parte del Instituto de Economía Social y Cooperativismo (INDESCO) de la Universidad Cooperativa de Colombia, lo mismo que de una editorial Argentina y una venezolana, el libro «Instituciones de Derecho Cooperativo, Mutual y Solidario», .

\section{b) Capacitación}

El IIDARA ha llevado a cabo cursos para directivos y asociados interesados, para promotores gubernamentales, para juristas y asesores. Hasta la fecha ha diseñado e impartido cursos de actualización, profundización y ampliación en distintas disciplinas del cooperativismo (promoción, dirección, gestión y legislación) para unos 400 profesionales y técnicos, funcionarios públicos y asociados de cooperativas de la zona occidental del país (Mérida, Barinas, Portuguesa, Cojedes, Lara, Yaracuy y Táchira) y para fines del año en curso otras 200 personas estarán siguiendo dichos cursos.

Se trata un programa de educación continua para egresados universitarios y técnicos superiores, asociados de cooperativas o que se desempeñen en funciones de promoción, asesoría y control cooperativo. Se utiliza metodología semipresencial a distancia, con materiales didácticos interactivos en formato electrónico, especialmente diseñado por personal especializado, con tutoría personalizada vía internet y actividades presenciales con profesores animadores expertos, concentradas en pocas sesiones eminentemente participativas, dedicadas a la resolución de problemas, el intercambio de opiniones, asesorías y evaluaciones.

Actualmente se desarrolla un programa conjunto con el Parque Tecnológico de la Universidad de Oriente para la preparación de profesores que asuman la capacitación de los cooperativistas en esta amplia zona del país.

Se trabaja permanentemente en la revisión de los contenidos de los módulos de estudio de manera de mantenerlos actualizados a las cambiantes circunstancias, así como en su adecuación a la metodología interactiva.

\section{c) Asesoría}

En problemas legales y de gestión a algunas cooperativas de base (cooperativa de servicios agrícolas La Trinidad, cooperativa de servicios 
múltiples Corandes); asesoría constante en materia de Derecho Cooperativo a centrales y federaciones de cooperativas de Venezuela, a la Confederación Latinoamericana de Cooperativas y Mutuales de Trabajadores (Colacot) de Bogotá y a la Alianza Cooperativista Nacional (Alcona) de México.

\section{El Técnico Superior en cooperativismo y economía social}

En este momento consideramos que es imperativo abrir las actividades académicas de la Universidad hacia el resto de la economía social, tanto por la importancia que han alcanzado otros sectores del mismo (cajas y fondos de ahorro, cajas agrarias, institutos de previsión social, fondos de jubilaciones, bancos comunales y empresas comunitarias) como por el reflujo en la actitud del gobierno actual hacia las cooperativas como formas asociativas privilegiadas de su accionar que posiblemente las dejará huérfanas de apoyo estatal, si no es que - por el contrario- sean objeto de discriminación negativa por el sector público.

Comoquiera que los destinatarios naturales de los programas educativos son personas (generalmente con estudios de secundaria completos) que se encuentran trabajando en sus empresas y organizaciones sociales, o en sus actividades privadas articuladas a cooperativas, deberán ser atendidos por métodos no tradicionales de educación. De esta manera, las actividades presenciales — de duración reducida a lo indispensable- deben ser concentradas en horarios y en días ajustados a las disponibilidades de los participantes, normalmente, en horas y días no laborables y en sitios cercanos a sus domicilios.

Es menester que estos estudios tengan cobertura nacional (no hay ninguna otra oferta académica pública nacional seria en tal sentido) y sea eminentemente a distancia, utilizando la plataforma "Moodle» que tiene instalada la Universidad, de manera de atender las necesidades formativas del sector asociativo nacional.

Estamos conscientes de la necesidad de mejorar cada vez más los contenidos y metodología de las actividades de capacitación, de manera de llegar — gradualmente- a la creación de estudios superiores en la materia, acreditados profesionalmente con títulos universitarios, ampliarlas a nivel nacional y aumentar el número de participantes.

Reiteramos que el programa de formación debe ser teórico-práctico, tomando como punto de referencia la experiencia de los participantes, eminentemente participativo y con salidas progresivas al mercado de trabajo que sería el de las propias empresas y organizaciones sociales en donde vienen desempeñándose. No se trata de recibir jóvenes 
estudiantes sin vinculación alguna con el sector para convertirlos en actores importados de empresas asociativas, sino elevar las capacidades y destrezas de quienes vienes actuando en ellas, acreditándolos académicamente.

De tal manera que, ajustados a la normativa nacional, podría haber un primer lapso (de 4 semestres) para otorgar el título de Técnico de nivel medio, y con 2 semestres mas, se podría obtener el título de Técnico Superior Universitario. Más adelante, se estudiaría la posibilidad de la Licenciatura (4 semestres adicionales) e incluso, estudios de postgrado, de acuerdo a las necesidades del medio y en la medida del apoyo institucional recibido.

Se trata de unificar y compartir los escasos y dispersos - geográfica e institucionalmente- recursos humanos de alto nivel con que cuentan las universidades públicas del país (sumar fortalezas y oportunidades) dotados de adecuadas destrezas y capacidades en la materia, en un esfuerzo conjunto de investigación, capacitación y asesoría.

Por ello, en el corto plazo debe llegarse a un convenio con el Núcleo Universitario «Tovar» y el Centro de Investigaciones para el Desarrollo Integral Sustentable (Núcleo de Trujillo) de la Universidad de los Andes; la Escuela de Estudios Sociales de la Universidad de Oriente (Cumaná); el Programa de Ciencias del Agro y Mar de la Universidad Nacional Experimental de los Llanos Occidentales «Ezequiel Zamora» (Barinas) y el Centro de Extensión y Cooperativismo de la Universidad Centro occidental Lisandro Alvarado (Barquisimeto) para elaborar y ejecutar conjuntamente el proyecto de Técnico Superior en Cooperativismo y Economía Social.

\section{Conclusiones}

a) Los programas sobre cooperativas de la Universidad de los Andes se han localizado fundamentalmente en la Facultad de Ciencias Jurídicas y Políticas, aunque en sus inicios lo fue en la Facultad de Economía y Ciencias Sociales;

b) La investigación y capacitación de esta Universidad en materia de cooperativismo y economía social se ha orientado más hacia lo doctrinario y lo jurídico que a lo gerencial o administrativo, lo que se hace necesario revertir de manera inmediata;

c) La calidad y cantidad de monografías elaboradas en el IIDARA de la ULA sobre tópicos cooperativistas, así como la edición de la revista Cayapa por el Núcleo Trujillo y su financiamiento por el CDCHT, la colocan como la primera institución nacional en el área; 
d) La diversidad de oferta de programas de capacitación de recurso humano, así como la experticia del profesorado que tiene la Universidad de los Andes, la convierte en el principal referendo nacional sobre la materia;

e) La metodología interactiva semipresencial a distancia empleada en los programas de capacitación ha resultado positiva, y junto al punto anterior, han sido la clave para el alto número de personas que han participado en los mismos;

f) La Universidad no ha contribuido con el aporte financiero esencial que hubiesen requerido los programas asumidos (planta física, equipamiento, gastos operativos y personal fijo) por lo que se ha debido acudir como ingreso principal al cobro de matrícula o a convenios con otras instituciones, lo que ha constituido una debilidad permanente;

g) Los programas académicos de la Universidad en el área cooperativa no han logrado tener cobertura nacional; a lo más, a escala meso regional en la zona occidental del país, con algunas incursiones en la zona oriental;

h) La perspectiva de implantar estudios profesionales interactivos a nivel nacional en convenio con otras dependencias de la Universidad de los Andes, así como con otras universidades públicas nacionales, puede ser la clave para la permanencia y el desarrollo sostenido de esta iniciativa universitaria.

\section{Referencias}

Capítulo de Mérida (1971). Ponencia a la IX Convención de Fecoacreve, Valencia Daly-Guevara, J. (1967). Derecho Cooperativo, Caracas, UCV

García-Müller, A. (1973). El Rol de las Cooperativas en América Latina. El caso de Venezuela, Mérida, Universidad de los Andes

García-Müller, A. (2002). La Escuela virtual de la Economía Social, Nápoles, Comunicación al 24 Congreso Internacional del CIRIEC

Martínez, J. (1972). Las Cooperativas de Venezuela, Caracas, Gumilla

TEXIER, J-M. (1969-1970). Aspectos de la cooperación en Venezuela, Informaciones Cooperativas, año 45, № 4/69 y 1/70, Ginebra, Organización Internacional del Trabajo. Mérida, junio de 2008 